\title{
Autokinetic drifts and gaze deviation
}

\author{
H. W. LEIBOWITZ, C. L. SHUPERT, R. B. POST, and J. DICHGANS \\ Pennsylvania State University, University Park, Pennsylvania
}

\begin{abstract}
A new method for evaluating the resting direction of the eyes based on the use of afterimages was developed. With this technique, the effect of gaze deviations relative to the individually determined resting direction was investigated among naive subjects. Moderate gaze deviations have no effect on reports of autokinesis. Only at extreme deviations which approach anatomical limits is the direction of reported autokinetic movement biased in the direction of the deviation. The possible contribution of efferent and afferent mechanisms to autokinesis is discussed.
\end{abstract}

The apparent movement of a stationary point of light viewed in an otherwise dark environment, referred to as autokinesis (AK), was first reported by Alexander von Humboldt in 1799 (Levy, 1972; Royce, Carran, Aftanas, Lehman, \& Blumenthal, 1972). Although numerous mechanisms have been proposed to account for this phenomenon, there is no general agreement as to its origin. A number of investigators have studied the effect of gaze deviation on the direction of apparent motion (Charpentier, 1886; Holmgren, 1889; Carr, 1910; Adams, 1912; Gregory \& Zangwill, 1963; Vaegan, 1976; Picoult, Young, \& Matin, 1979), and have consistently reported that an AK stimulus viewed with gaze deviated from the primary position is reported to move in the same direction as the deviation. Two mechanisms have been proposed to account for such effects, both of which hypothesize that maintaining deviated gaze results in the directional bias. One mechanism relies on efferent motion perception theories. In accordance with reafference theory, as formulated by Helmholtz (1962, p. 234) and later elaborated by von Holst and Mittelstaedt (1950), it is assumed that voluntary motor commands simultaneously generate sensory expectations referred to as "efference copies" or "corollary discharges" (Sperry, 1950), which match and cancel the afference resulting from sense organ movement. If this were not the case, the organism would be unable to distinguish afference resulting from stimulation of stationary sense organs

This research was supported by Grants MH08061, from the National Institutes of Health, and EY03276, from the National Eye Institute, and by a Senior Scientist Award to H. W. Leibowitz from the Alexander von Humboldt Foundation for study at the Department of Neurology, University of Freiburg. The authors are grateful to Jane Raymond and Muzafer Sherif for assistance in the preparation of the manuscript. Current addresses are: $\mathbf{R}$. B. Post, Department of Ophthalmology, University of California, Davis, California 95616; J. Dichgans, Department of Neurology, University of Tübingen, Tübingen, West Germany. Reprint requests should be sent to: H. Leibowitz, Moore Building, Pennsylvania State University, University Park, Pennsylvania 16802. (exafference) from the self-produced afference resulting from movement of the sense organs (reafference). The perceived movement of an object that is tracked so that the retinal image remains relatively motionless is assumed to be mediated by the uncanceled efference copy. In the case of an eccentrically located AK stimulus, it is assumed that the eyes will tend to drift back to the resting direction unless this tendency is counteracted by the activation of extraocular muscles that oppose the direction of drift. This voluntary effort would, according to reafference theory, be accompanied by an efference copy, but since the muscular activity is directed at maintaining fixation on the AK stimulus, no reafference (movement of the retinal image) follows. As a result, the uncanceled efference copy is perceived as illusory movement of the fixated stimulus in the direction of the voluntary effort. ${ }^{1}$

In addition to this efferent explanation, afferent mechanisms have also been proposed to account for the reported effects of gaze deviation on the direction of AK. Specifically, it has been suggested that, during the attempt to maintain eccentric fixation, small drifts of the eyes occur in the direction of the resting direction. This results in retinal image motion of the fixated stimulus and stimulation of afferent motion-analyzing mechanisms (Matin \& MacKinnon, 1964).

Despite the fact that the effect of gaze deviation on AK is well established in the literature, it was decided to reanalyze the relationship between gaze deviation and AK drifts. There are two bases for reinvestigating this relationship.

\section{Suggestion}

It has been recognized since the classic studies of Sherif (1935) that suggestion plays a major role in reports of AK. In the course of investigating gazedeviation effects in our laboratory, we discovered that subjects who were aware of the hypothesis that gaze deviation should bias AK in the direction of the effort produced data that conformed with the theory. 
Contrary results, however, were obtained from naive subjects, who reported random AK movement for moderate gaze deviation (up to $22.5 \mathrm{deg}$ ). Moreover, if naive subjects were made aware of the theory, their subsequent responses were biased in the expected direction.

As a result of these observations, only naive subjects were tested in the present experiments, in contrast to many previous investigations of gaze deviation. A detailed description of studies in which expectations were systematically manipulated and a theoretical discussion of possible mechanisms underlying this effect have been reported elsewhere (Leibowitz, Shupert, Post, \& Dichgans, in press).

\section{The Resting Direction of the Eyes}

If maintaining deviated gaze is a factor in AK, it is necessary to know where the eyes are normally directed in the absence of a fixation stimulus. Knowledge of this "resting direction of the eyes" permits specification of the direction and magnitude of gaze deviation. Consistent with this reasoning, Vaegan (1976) identified a position of the stimulus relative to the observer at which the reported direction of $\mathrm{AK}$ is random. He referred to this as "the physiological position of rest and phoria." Stimuli located at other positions were reported to move in the direction of the gaze deviation presumably as a result of the effort required to maintain a deviated gaze.

In the present study, a new method for determining the resting direction of the eyes was developed. This technique, which assumes that the localization of an afterimage corresponds to its retinal location, is based on an observation by Troland (1917), that a faded afterimage is reactivated by changes in background luminance. A foveal afterimage is produced by a photoflash, after which the subject sits quietly in the dark and makes no effort to move the eyes. After the afterimage has faded, it is reactivated by dimly illuminating a screen in front of the subject, who is requested to mark the apparent position of the afterimage. After a few trials, this procedure exhibits high reliability for most subjects. This procedure, unlike Vaegan's method, is independent of reports of AK.

The considerable intersubject variability in the resting direction is indicated by the scatterplot in Figure 1 for 93 naive observers. In general, the resting direction is below the horizontal plane of the eyes and left of the median sagittal plane. Intersubject variability in this particular oculomotor resting position is not entirely unexpected in view of previous reports that the oculomotor adjustments of accommodation (Leibowitz \& Owens, 1978) and convergence (Owens \& Leibowitz, 1976, 1980) both demonstrate marked intersubject variability in their resting positions. The origin and significance of this variability will not be discussed here. However, it is clear that individual variability in the resting direction is

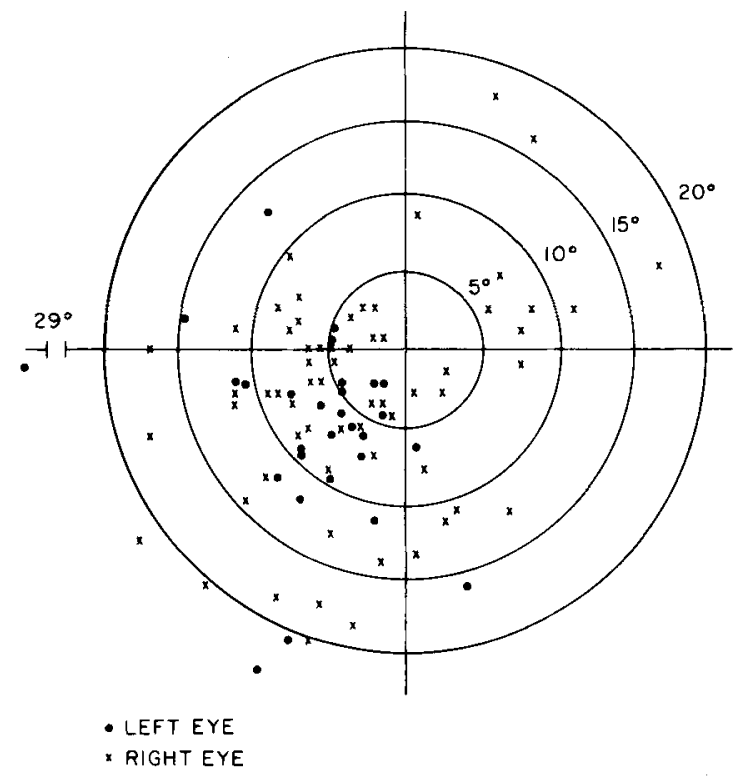

Figure 1. Scatterplot of the direction of gaze in the dark for 93 subjects.

marked and could be important in experimental studies of the effect of gaze deviation on AK. Given this large intersubject variability in the resting direction, the individually determined values of the resting direction were used as a null position in all studies of gaze deviation.

Two studies are reported. In the first, AK data were obtained at the resting direction and for $22.5 \mathrm{deg}$ of eccentricity at the four cardinal directions. In the second study, the AK stimulus was presented in the horizontal meridian at eccentricities up to $60 \mathrm{deg}$ from the individually determined resting direction.

\section{EXPERIMENT 1}

\section{Method}

Eleven males and 19 females, who were naive to the purpose of the experiment, participated in the experiment. The subjects were recruited from introductory psychology courses and received course credit in compensation.

The subjects were tested individually. At the beginning of the session, the subject was seated at a chinrest $50 \mathrm{~cm}$ in front of a screen, which filled the entire field of view. The nondominant eye (as determined by a sighting technique) was patched, and the subject was instructed to fixate a point on a photoflash that was masked in such a way as to generate a rectangular afterimage, subtending $4.5 \times 2.25 \mathrm{deg}$, with an unstimulated circular area, $2.25 \mathrm{deg}$ in diameter, in the center. After the flash was activated, the room lights were extinguished and the subject allowed the afterimage to fade. When fading was complete, the subject illuminated the screen with a flashlight and marked the position of the renewed afterimage. Approximately seven such positions were determined. The median of the horizontal and vertical distributions of the marks was used as the subject's resting direction. Following the location of the resting direction, the subject was asked to observe the AK movement of a light-emitting diode affixed to the screen at one of five positions: at the subject's resting direction and at $22.5 \mathrm{deg}$ directly up, down, right, or left of the resting 
direction. Three 20 -sec trials were carried out for each position of the diode, and positions were presented in random order. On each trial, the subject rested for $20 \mathrm{sec}$ with eyes closed, following which the experimenter gave a verbal command to start observation. The subject observed the light-emitting diode for $20 \mathrm{sec}$ while listening to a metronome ticking at $1 \mathrm{~Hz}$, and then verbally reported the number of seconds of AK movement observed in each of eight directions: up, down, left, right, and the four obliques. ${ }^{2}$

\section{Results}

The data, presented as the percentage of time directional AK movement was reported, are described in Figure 2. For each stimulus position, the number of seconds of movement reported to the north, east, south, or west was summed across all trials. A $5 \times 4$ (stimulus position $\times$ reported direction of movement) analysis of variance for repeated measures was performed. A significant main effect was found for reported direction of movement $[F(3,87)=9.05, p<$ $.01]$, and Newman-Keuls analysis indicated that north movement was reported more frequently than any other direction. Furthermore, stimulus position and reported direction of movement were found to interact significantly $[F(12,348)=3.18, p<.01]$. A Newman-Keuls analysis indicated that north movement was seen significantly less at east and west positions of the stimulus than for other stimulus positions. No other reported direction differed across stimulus positions.

In order to more systematically investigate the role of gaze deviation, a subsequent study was conducted in which the stimulus was located $22.5,40$, or $60 \mathrm{deg}$ from the resting direction along the horizontal me-

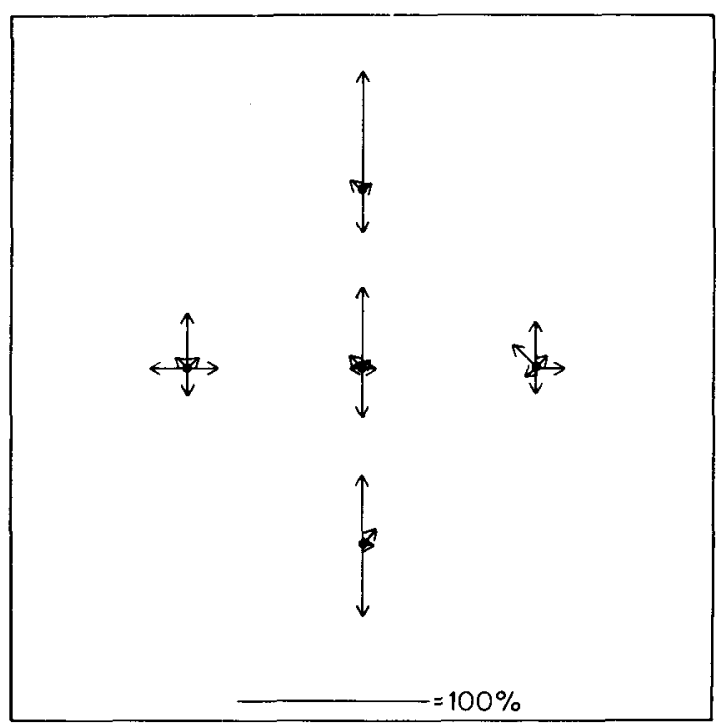

Figure 2. The effect of gaze deviation of 22.5 deg on the direction of autokinetic movement. The central position corresponds to the individually determined resting position. The other positions represent gaze deviations of $22.5 \mathrm{deg}$. The length of the vection is proportional to the percentage of time directionally biased autokinetic movement was observed. ridian. A 60-deg deviation is not possible with movement of the eyes alone; rather, it represents a combination of maximum eye deviation plus some head movement.

\section{EXPERIMENT 2}

\section{Method}

Thirty adult volunteers (19 females, 11 males) participated. All had observed AK movement prior to participating but were naive to the purpose of the experiment. The resting direction of the dominant eye was determined for each subject as in Experiment 1. The experiment consisted of $1820-\mathrm{sec}$ trials carried out in blocks of 3 trials at each of six positions of the AK stimulus: at the resting direction and at $22.5 \mathrm{deg}$ to the right, $22.5 \mathrm{deg}$ to the left, $40 \mathrm{deg}$ to the right, $40 \mathrm{deg}$ to the left, and $60 \mathrm{deg}$ to the right or left (to the left for left-eye-dominant subjects and to the right for right-eye-dominant subjects). AK movement was recorded as in Experiment 1.

\section{Results}

The average number of seconds of AK movement reported in each of eight directions as a percentage of the total movement observed at the seven stimulus positions is presented in Figure 3. The results indicate that the tendency to report $\mathbf{A K}$ in the direction of gaze deviation is unaffected by a deviation of $22.5 \mathrm{deg}$ from the resting direction as previously reported. There is some gaze deviation bias at $\mathbf{4 0} \mathrm{deg}$. Only at $60 \mathrm{deg}$, which involves a maximum eye deviation plus some head rotation, does AK tend to be strongly directionally biased in the direction of gaze deviation.

A statistical analysis of the data confirms the trends present in the diagram. Data for right-eyedominant subjects $(n=24)$ were grouped separately from those for left-eye-dominant subjects $(n=6)$, and the amount of east or west movement reported at each stimulus position was summed across all trials. A $2 \times 6 \times 2$ (eye-dominance group $\times$ stimulus position $\times$ reported direction of movement) analysis of variance with repeated measures on the last two factors was performed and yielded the following significant effects. Significantly more AK movement was reported for stimulus positions 60 deg eccentric to the resting direction than for all other stimulus positions $[F(5,140)=5.63, p<.01]$. In addition, subjects in the right-eye-dominant group reported significantly more east movement than west, while the left-eyedominant group reported significantly more west movement than east $[F(1,28)=8.25, p<.01]$. This is expected, because right-eye-dominant subjects viewed the stimulus $60 \mathrm{deg}$ eccentric to the resting direction to the right of the resting direction, while left-eyedominant subjects viewed it to the left of the resting direction. Both eccentricity and direction $[F(5,140)=$ $2.70, p<.05]$ and eccentricity, direction, and group $[F(4,140)=4.10, p<.01]$ were found to interact significantly. A Newman-Keuls analysis of these effects indicates that, while the amount of east and west 


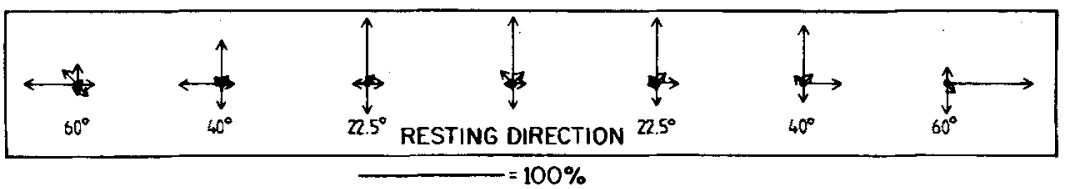

Figure 3. The effect of gaze deviation in the horizontal meridian on the direction of autokinetic movement. The length of the vector is proportional to the percentage of time directionally biased autokinetic movement was observed.

movement reported for stimuli placed at the resting

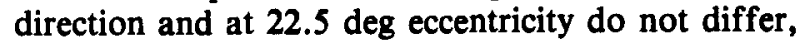
reports in the predicted direction for stimuli placed 40 or $60 \mathrm{deg}$ eccentric to the resting direction.

\section{DISCUSSION}

The present results indicate that the role of gaze deviation in AK is minor. Only deviations that approach anatomical limits result in biased AK movement.

With respect to the mechanism subserving this phenomenon, there are two categories of possible explanations:

Efferent-Whenever the pursuit system is activated an efferent motion signal is simultaneously generated. This signal, referred to variously as efference copy (von Holst \& Mittelstaedt, 1950) or corollary discharge (Sperry, 1950), serves to mediate object motion perception during visual pursuit. In the case of voluntary pursuit, even though the retinal image is nearly stationary, the neural signal involved in tracking subserves motion perception. The efferent interpretation of the effect of gaze deviation argues that maintenance of extreme gaze deviations also requires a voluntary effort of the pursuit system and that the associated efferent motion signal is responsible for the illusory motion. Thus, the effort required to maintain deviated gaze produces an efferent motion signal, with the result that illusory motion is perceived in the direction of the effort.

Afferent-The eyes are not perfectly stable during fixation (see, e.g., Steinman, 1965), so that retinal image motion can occur as a consequence of involuntary losses of fixation. In afferent interpretations of AK movement, this motion is presumed to stimulate directionally specific motion analyzers. In the case of deviated gaze, it is assumed that the losses of fixation are increasingly biased toward the resting direction of the eyes as a function of eccentricity. As a result, those motion analyzers responsible for signaling object motion in the opposite direction are stimulated, leading to the perception of target motion away from the resting direction. Alternatively, it can be hypothesized that extraocular muscle stretch receptors could provide a signal indicating the effort involved in maintaining eccentric gaze. Misinterpretation of this signal as arising from a pursuit move- ment could result in illusory motion of the fixated stimulus.

Both of these explanations are consistent with other examples of illusory motion. For example, the oculogyral illusion is assumed to result from the effort required to preserve fixational stability by suppressing vestibular nystagmus generated by the vestibuloocular reflex (Whiteside, Graybiel, \& Niven, 1965). Similarly, the apparent movement of a fixated point of light in the dark during head translation is assumed to result from the effort required to oppose or supplement the smooth compensatory eye movements associated with lateral acceleration of the head (Post \& Leibowitz, 1982).

On the basis of both the present data and previous reports, it is not possible to decide among these alternatives. For example, as extraocular muscle is progressively weakened by curarization, it would be expected that, for a given eccentricity, the effort required to maintain deviated gaze would increase, while activation of the stretch receptors would decrease. Matin (Note 1) observed that the illusory motion of eccentrically located stimuli increases with the level of paralysis. These observations are consistent with both afferent and efferent interpretations, since both the pursuit effort required to maintain fixation and the amount of retinal slip would increase with increasing paralysis.

However, evidence for an efferent interpretation is provided by studies of eye movements. It is well known that AK decreases with increasing target size (Royce et al., 1966). In contrast to this finding, Steinman (1965) has reported that the pattern of small eye movements is little influenced by target size. This pattern of results is inconsistent with an afferent interpretation, since the amount of retinal slip is unrelated to target size. It is likely that the decrease in AK with large stimuli results from the increasing contribution of reflexive gaze stabilization mechanisms that are known to be activated more with larger contour (Dichgans, Nauck, \& Wolpert, 1973). With increasing reflexive stabilization, correspondingly less voluntary effort is required to maintain fixation. Further support for this notion comes from the data of Westall and Aslin (1982), who have observed the pattern of small eye movements occurring both during fixation of a target that exhibits AK movement and during fixation of one 
that does not. Their findings show no difference in the frequency and amplitude of these movements between these two conditions. It is therefore unlikely that retinal image motion plays a large role in AK movement.

\section{REFERENCE NOTE}

1. Matin, L. Personal communication, 1982.

\section{REFERENCES}

Adams, H. F. Autokinetic sensations. Psychological Monographs, 1912, 14, 1-45.

CArr, H. A. The autokinetic sensation. Psychological Review, $1910,17,42.75$.

Charpentier, A. Sur une illusion visuelle. Comptes Rendues Academie des Sciences, Paris, 1886, 102, 1155-1157.

Dichgans, J., Nauck, B., \& Wolpent, E. The influence of attention, vigilance, and stimulus area on optokinetic and vestibular nystagmus and voluntary saccades. In V. Zikmund (Ed.), The oculomotor system and brain function. London: Butterworth, 1973.

Gregory, R., \& Zanowili, O. L. The origin of the autokinetic effect. Quarterly Journal of Experimental Psychology, 1963, 15, 252-261.

Helmholtz, H. Physiological optics II. (J. P. C. Southall, trans.). New York: Dover, 1962.

Holmoren, S. Studien über die elementaren Farbenempfindungen. Skandinavisches Archiv fur Physiologie, 1889, 1, 152-183.

Leibowitz, H. W., \& Owens, D. A. New evidence for the intermediate position of relaxed accommodation. Documenta Ophthalmologica, 1978, 46, 133-147.

Leibowitz, H. W., Shupert, C. L., Post, R. B., \& Dichgans, J. Expectation and autokinesis. Perception \& Psychophysics, in press.

LEvY, J. Autokinetic illusion: A systematic review of theories, measures, and independent variables. Psychological Bulletin, $1972,78,457-474$.

Matin, L., \& MacKinnon, G. E. Autokinetic movement: Selective manipulation of directional components by image stabilization. Science, 1964, 143, 147-148.

OWens, D. A., \& Leibowitz, H. W. Oculomotor adjustments in darkness and the specific distance tendency. Perception \& Psychophysics, 1976, 20, 2-9.

Owens, D. A., \& Leibowitz, H. W. Accommodation, convergence, and distance perception in low illumination. American Journal of Optometry and Physiological Optics, 1980, 57, 540550.

Picoult, E., Young, D., \& Matin, L. Eye movement, gaze deviation, and autokinesis. Investigative Ophthalmology and Visual Science, 1979, Supplement, 102.
Post, R. B., \& Leibowitz, H. W. The effect of convergence on the vestibulo-ocular reflex and implications for perceived movement. Vision Research, 1982, 22, 461-465.

Royce, J. R., Carran, A. B., Aftanas, M., Lehman, R. S., \& Blumenthal, A. The autokinetic phenomenon: A critical review. Psychological Bulletin, 1966, 65, 243-260.

SHE RIF, M. A study of some social factors in perception. Archives of Psychology, 1935, 187, 1-60.

SPERRY, R. W. Neural basis of the spontaneous optokinetic response produced by visual inversion. Journal of Comparative Physiological Psychology, 1950, 43, 482-489.

Steinman, R. M. Effect of target size, luminance and color on monocular fixation. Journal of the Optical Society of America, $1965,55,1158-1165$.

Troland, L. T. Preliminary note: The influence of changes of illumination upon afterimages. American Journal of Psychology, 1917, 28, 497-503.

VEAGAN. The position of random autokinetic movement and the physiological position of rest are frequentiy stable and identical. Perception \& Psychophysics, 1976, 19, 240-245.

von Holst, E., \& Mittelstaedt, H. Das Reafferenzprinzip, Wechselswirkungen zwischen Zentralnervensystem und Peripherie. Naturwissenschaften, 1950, 37, 464-476.

Westall, C., \& Astin, R. Fixational eye movements and autokinesis in amblyopia. Investigative Ophthalmology and Visual Science, 1982, 22, 89. (Suppl.)

Whiteside, T. C. D., Graybiel, A., \& Nrven, J. I. Visual illusions of movement. Brain, 1965, 88, 193-210.

\section{NOTES}

1. Other phenomena involving illusory movement can be parsimoniously interpreted within this framework. For an analysis of the apparent movement of a single light viewed in the dark while making translatory head movements, see Post and Leibowitz, 1982.

2. In many previous studies, particularly the classical investigations of Sherif (1935), AK was evaluated primarily in terms of the extent of magnitude of the illusory movement. Extent of movement is not only susceptible to response bias, because there is no physical reference, but estimates of magnitudes are also complicated by the fact that the apparently moving object does not change location. For these reasons, AK was evaluated in the present study in terms of the duration of illusory movement observed in a given direction. To provide a physical reference, a metronome was activated at $1 \mathrm{~Hz}$. The subject reported the number of seconds of AK movement.

(Manuscript received September 21, 1981; revision accepted for publication January $28,1983$. 